\title{
Priority monism and essentiality of fundamentality: a reply to Steinberg
}

\author{
Matteo Benocci ${ }^{1}$ (D)
}

Published online: 16 September 2016

(c) The Author(s) 2016. This article is published with open access at Springerlink.com

\begin{abstract}
Steinberg has recently proposed an argument against Schaffer's priority monism. The argument assumes the principle of Necessity of Monism, which states that if priority monism is true, then it is necessarily true. In this paper, I argue that Steinberg's objection can be eluded by giving up Necessity of Monism for an alternative principle, that I call Essentiality of Fundamentality, and that such a principle is to be preferred to Necessity of Monism on other grounds as well.
\end{abstract}

Keywords Priority monism $\cdot$ Dependence $\cdot$ Fundamentality $\cdot$ Schaffer, Jonathan

Priority monism is the thesis, defended by Schaffer in a series of articles (2007, 2010a, b, 2013), that the cosmos is a fundamental whole, and that all subcosmic objects depend on it. ${ }^{1}$ Steinberg has shown that, given certain assumptions, it follows from priority monism that no whole depends on its proper parts. This consequence is highly implausible, ${ }^{2}$ hence the argument seems to qualify as a reductio ad absurdum of priority monism. Steinberg's argument relies on three

\footnotetext{
1 Actually, Schaffer has often stated priority monism in terms of grounding, adopting the concept of grounding as primitive and without clarifying its relationship with the akin concept of ontological dependence. I agree with Steinberg that Schaffer's terminology is misleading, because usually grounding is categorially restricted to facts (Steinberg 2015, p. 2027). For this reason, I will phrase the discussion in terms of dependence.

2 Indeed, Schaffer himself claims that priority monism is compatible with the view that some subcosmic composites depend on their proper parts, though being ultimately dependent on the whole cosmos (Schaffer 2010a, p. 44).
}

Matteo Benocci

m.benocci@pgr.reading.ac.uk

1 Department of Philosophy, University of Reading, Reading RG6 6AA, UK 
main assumptions: the first assumption is Necessity of Monism, namely the claim that, if priority monism is true, then it is necessarily true. The second assumption is what Steinberg calls Internality of Dependence: for any objects $x$ and $y$, if $x$ depends on $y$ in some possible world, then $x$ depends on $y$ in every possible world where both $x$ and $y$ exist. The third assumption is a principle of Isolation: for any composite $x$ which exists at the actual world, there is a possible world where the only objects which exist are $x$ and its parts. The argument is the following:

Assume that (A) priority monism is true and suppose for reductio that (S) some composite $c$ depends on one of its proper parts $p$. By Isolation, there is a world $w$ that includes only $c$ and its parts, among them $p$. By Internality of Dependence, since $c$ and $p$ exist at $w, c$ depends on $p$ at $w$. By (A) and Necessity of Monism, at $w$, the cosmos is the one and only basic concrete object. Since, at $w, c=$ the cosmos, $c$ does not depend on anything concrete at $w$, in particular not on $p$. Contradiction! We may thus deny our supposition (S) and discharge our assumption (A): if priority monism is true, no composite depends on any of its parts. (2015, p. 2026)

Steinberg convincingly argues that there is no reasonable way to deny Isolation or Internality of Dependence (2015, pp. 2027f.). The remaining option to elude this argument would be to reject Necessity of Monism, but Steinberg also argues that this move is not viable (2015, pp. 2026f.), since Schaffer himself endorses that principle (2010a, p. 56) and employs it to support priority monism.

In this paper, I will argue that Steinberg's objection can be eluded by giving up Necessity of Monism for an alternative principle, that I will call Essentiality of Fundamentality: necessarily, for every $\operatorname{cosmos} c$, if $c$ is fundamental then $c$ is essentially fundamental. I will show that this principle, if properly worked out, is preferable to Necessity of Monism on other grounds as well, so replacing Necessity of Monism with it would not be an ad hoc move. Finally, I will argue that the cost of this move for Schaffer's argumentative strategy is smaller than Steinberg suggests, and ultimately worth paying. At the end of the day, friends of priority monism have good reasons to give up Necessity of Monism for Essentiality of Fundamentality.

In order to state Essentiality of Fundamentality and contrast it with Necessity of Monism, one needs to clarify the relationships between the concept of cosmos and that of possible world. I define a cosmos as a causally isolated system, i.e. a system which cannot interact with any system mereologically disjoint from it. Putting aside the complex issues about the ontological status of possible worlds, a possible world can be roughly defined as a complete possible state of reality ${ }^{3}$ : with this in mind, a cosmos is just one of the objects which exist at a possible world, though an object of a peculiar sort. Like subcosmic objects, cosmoi exist at many possible worlds, and are subject to transworld identity and distinctness: there are possible worlds $w$ and $w^{\prime}$, with a $\operatorname{cosmos} c$ existing at $w$ and a $\operatorname{cosmos} c^{\prime}$ existing at $w^{\prime}$, such that $c \neq c$ '. Particularly, there are possible worlds where there are cosmoi distinct from this

\footnotetext{
3 Incidentally, this sounds like an ontologically neutral version of Plantinga's definition of a possible world as a maximal consistent state of affairs (Plantinga 1974).
} 
cosmos, the one we are part of. According to the present definition of "cosmos", there are also possible worlds where many cosmoi exist, since it is possible that there are many causally isolated systems. ${ }^{4}$ On the other hand, in the empty possible world, i.e. the possible world where no object exists, there is no cosmos at all. We know that in the actual world there is at least one cosmos, namely the cosmos we are part of, but that there are other cosmoi too is an epistemic possibility we should stay agnostic about. $^{5}$

A cosmos is fundamental iff (i) it does not depend on any object, and (ii) it is prior to all its proper parts or, to put it in a different way, all its proper parts depend on it. A cosmos is pluralistic iff it is not fundamental. Priority monism, in its minimal formulation, is the claim that this cosmos is fundamental. Within the present framework, Necessity of Monism can be restated as the claim that, in every possible world $w$, every cosmos (which exists at $w$ ) is fundamental at $w$. Essentiality of Fundamentality is to be formulated in the following way: for every possible world $w$, for every $\operatorname{cosmos} c$, if $c$ is fundamental at $w$ then $c$ is fundamental in all the possible worlds where it exists. Differently from Necessity of Monism, Essentiality of Fundamentality does not rule out that in some possible worlds there is a pluralistic cosmos: the conjunction of priority monism together with Essentiality of Fundamentality only entails that this cosmos is essentially fundamental, i.e. is fundamental in all the possible worlds where it exists.

What is the support for this view? A motivation for Essentiality of Fundamentality can be worked out by embracing the claim, defended by Bigelow et al. (1992), that the cosmos belongs to a certain natural kind which is essential to it. ${ }^{6}$ Let us formulate this view in terms of possible worlds: in every possible world, every cosmos belongs to a natural kind which is essential to it. The natural kind a cosmos belongs to is, arguably, defined by certain natural properties instantiated by that cosmos as a whole and by a related set of laws of nature: cosmic properties and laws are not independent, but come together, since a cosmos displays its properties by developing according to certain laws. With this in mind, it is reasonable to include

\footnotetext{
4 It is worth noticing that Schaffer actually defines the cosmos without invoking any causal notion, as that object of which all actual objects are part (Schaffer 2010a, p. 33), which implies that there is exactly one cosmos. Nevertheless, I consider the present definition in terms of causal isolation to be superior. First, it is closer to the traditional connotation of the term, which conveys the idea of an ordered and unified whole (and actually comes from the Ancient Greek word "кó $\rho \mu \varsigma$ ", which is usually translated by "order" or "harmony"). Therefore, causal unity and isolation should be included in the very definition of the term. Furthermore, Schaffer strongly relies on modern physics and cosmology to defend priority monism, and scientific cosmology treats the cosmos mainly as an integrated causal system. In conclusion, my definition is closer to the spirit of priority monism than the definition employed by Schaffer himself.

5 Actually, whether there are or not cosmoi other than this one is an empirically undecidable matter, because according to the present definition another cosmos would be causally isolated from the one we inhabit, but any observation requires an appropriate causal connection between the observer and the observed. It is also worth noticing that, under the present definition of "cosmos", the physical system that theoretical physicists call multiverse would still be one cosmos, not a collection of many cosmoi, since its branches are supposed to be causally related: if the multiverse hypothesis was shown to be true, that would not count as a scenario in which we discover that there are many cosmoi, but as a scenario in which we discover that this cosmos has a certain kind of physical structure.

6 Bigelow, Ellis and Lierse actually use the word "world", by which they intuitively refer to the sort of system that I call "cosmos".
} 
in the inventory of essential features of a cosmos its structure too, intended as the priority hierarchy which obtains between its (proper or improper) parts: whether certain parts of a cosmos are prior to its other parts or dependent on them is an essential feature of that cosmos. To say it with a slogan, a cosmos where dependence works in a different way is another cosmos. This move is by no means artificial: actually, it is suggested by the very argumentative strategy employed by Schaffer to support priority monism. For instance, his argument from quantum mechanics rests on the premise that the cosmos is an entangled system: that the cosmos is an entangled system entails that the cosmos is fundamental, since an entangled system is prior to its proper parts (Schaffer 2010a, pp. 52ff.). Within the view I am outlining, being an entangled system is an obvious candidate as an essential property of this cosmos, and the properties entailed by an essential property are essential too: in this case, if a cosmos is essentially an entangled system, then it is essentially fundamental. In conclusion, it seems that the natural properties of a cosmos, the laws according to which it develops over time and its structure form an organic package deal: properties and laws necessarily come together, and entail a certain kind of priority hierarchy. Once that structure has been rightfully included among the essential features of a cosmos, Essentiality of Fundamentality follows: if a cosmos is fundamental, then it is essentially so.

Essentiality of Fundamentality can be seen as a replacement for Necessity of Monism since it preserves Schaffer's intuition that priority monism is somehow a metaphysical law, because it is concerned with the essential structure of the cosmos, not with its accidental features. With this in mind, I claim that Essentiality of Fundamentality is to be preferred to Necessity of Monism, since it avoids the arbitrary restrictions of the space of metaphysical possibilities imposed by the latter: after all, what is absurd about a pluralistic cosmos? Even if this cosmos is essentially fundamental, the possibility of a cosmos of a different kind with a pluralistic structure still seems warranted. Schaffer has defended Necessity of Monism by embracing loose slogans, like the claim that "... metaphysical possibilities hold fixed how grounding works" (Schaffer 2010a, p. 63), or the claim that priority monism is a metaphysical law, and metaphysical laws "... govern what grounds what" (Schaffer 2010a, p. 56). To be sure, there is some necessary, analytic truth about dependence, like the principles of irreflexivity, asymmetry, and transitivity, and maybe there is also some synthetic principle, like that of wellfoundedness, which states that endless dependence chains are impossible (Schaffer 2010a, pp. 37, 62). That said, there is no reason why a certain priority hierarchy should obtain in all possible worlds, no matter which kinds of objects exist. Essentiality of Fundamentality has the advantage of avoiding such arbitrary restrictions of the space of metaphysical possibilities.

Given the distinction above between cosmoi and possible worlds, Steinberg's application of Isolation and Internality of Dependence is still straightforward: a composite $a$ which is subcosmic at the actual world, is a cosmos at some possible worlds, since in a possible world where only $a$ and its parts exist $a$ is ipso facto causally isolated. If in the actual world $a$ depends on its proper parts, then in those possible worlds where $a$ is a cosmos, $a$ is a pluralistic cosmos. Nevertheless, once Necessity of Monism has been abandoned for Essentiality of Fundamentality 
Steinberg's reductio fails in its original form. On the other hand, it cannot be reconstructed by appealing to Essentiality of Fundamentality, because of the modal differences between a cosmos and its denizens. The quickest way to see that is to invoke the principle of necessity of distinctness (Kripke 1980, p. 114): for any $x$ and $y$, if $x$ and $y$ are numerically distinct, then it is impossible that $x$ is identical to $y$, i.e. there is no possible world where $x$ is identical to $y .^{7}$ This cosmos and a denizen of it are numerically distinct, so it is impossible that they are identical: given a subcosmic object $a$, it is possible that $a$ is a cosmos, but it is not possible that $a$ is identical to this cosmos. Therefore, the actual cosmos being essentially fundamental (which follows from the conjunction of priority monism and Essentiality of Fundamentality) and the possibility of an actual subcosmic composite $a$ being a pluralistic cosmos do not entail the possibility of $a$ being a fundamental cosmos, so they do not contradict Internality of Dependence. The reductio is eluded.

That said, the necessity of identity and the necessity of distinctness are not uncontroversial, so it is worth stressing that there are also other modal differences between a cosmos and its denizens which make Essentiality of Fundamentality unsuited for Steinberg's reductio. Every subcosmic composite is either an integrated whole or a mere aggregate. Integrated wholes like living organisms essentially belong to a certain natural kind, which is different from that of the cosmos they inhabit: for instance, Socrates is essentially a human being, whereas the cosmos is not. It is possible that Socrates is a $\operatorname{cosmos}^{8}{ }^{8}$ but not that he is identical to this cosmos. Therefore, the possibility of Socrates being a cosmos does not contradict Internality of Dependence. One can make analogous considerations in the case of mere aggregates, like a heap of sand: a heap is, arguably, mereologically fragile, because it has its parts essentially. On the other hand, this cosmos is mereologically flexible, since it might be composed of subcosmic objects other than those it is actually composed of. Again, even if it is possible that a heap is a cosmos, it is not possible that it is identical to this cosmos. In any case, priority monism, Essentiality of Fundamentality and the possibility of an actual subcosmic composite being a cosmos do not entail that there are possible worlds which contradict Internality of Dependence.

Steinberg might reply, as an ad hominem point, that eluding his objection by replacing Necessity of Monism with Essentiality of Fundamentality is a Pyrrhic victory, because Necessity of Monism is required to build Schaffer's arguments for priority monism: indeed, Steinberg suggests en passant that “... at the very least, the priority monist would lose significant argumentative ground by giving up Necessity of Monism” (2015, p. 2027). Actually, only two of Schaffer's arguments, namely the argument from the possibility of gunk and the argument from the possibility emergence, rely on what I will briefly call Modal Exclusiveness: either

\footnotetext{
7 In a footnote, Kripke shows that the necessity of distinctness can be proven in a way analogous to that of the necessity of identity: "Suppose $X \neq Y$; if $X$ and $Y$ were both identical to some object $Z$ in another possible world, then $X=Z, Y=Z$, hence $X=Y$ " (Kripke 1980, p. 114).

8 Actually, Steinberg acknowledges that Isolation might be false for living organisms (2015, p. 2028), since it conflicts with Kripke's thesis of the necessity of origin (Kripke 1980, pp. 112f.). However, here I am granting this point at least for the sake of argument.
} 
priority monism is necessarily true, or pluralism is necessarily true (Schaffer 2010a, p. 56). ${ }^{9}$ Necessity of Monism follows logically from Modal Exclusiveness: thus, the rejection of the former entails the rejection of the latter. I will show that the argument from the possibility of gunk can be vindicated even without that principle. On the other hand, the argument from the possibility of emergence cannot be vindicated, but this represents a negligible loss.

In Schaffer's formulation, the argument from the possibility of gunk assumes the metaphysical possibility of gunk: the existence of portions of atomless matter is at least metaphysically possible. Schaffer argues that pluralists can accommodate this metaphysical possibility in three ways: (1) accepting that an endless dependence chain is possible, (2) maintaining that the basic objects are some subcosmic composites, or (3) granting that pluralism is false at gunky possible worlds. (1) is incompatible with the principle of well-foundedness, (2) is arbitrary, and (3) is incompatible with Modal Exclusiveness. Therefore, pluralism is false, and, by Modal Exclusiveness, priority monism is (necessarily) true (Schaffer 2010a, pp. 61ff.). Once Necessity of Monism and, together with it, Modal Exclusiveness, has been rejected, the argument from the possibility of gunk fails, as long as it relies on the metaphysical possibility of gunk. Nonetheless, it can be reformulated as an argument from the epistemic possibility of gunk. As far as we know, this cosmos may be (entirely or partly) gunky: Schaffer himself stresses that there are "... scientifically serious, empirically open hypotheses..." which posit an endless descent toward lower and lower levels (Schaffer 2010a, p. 62), like the infinite regression of sub-electron particles hypothesized by Dehmelt (1989), or the infinite quantum fields regression hypothesized by Georgi (1989). Pluralists can accommodate such eventualities only by endorsing one of the options (1) and (2) above, but those are troublesome. Therefore, pluralism seems to rule out gunk hypotheses, imposing an armchair, a priori constraint on an open empirical question, whereas priority monism does not. In this respect, the latter has an advantage over the former, since it is not threatened by the eventuality of "bad news" from physics. ${ }^{10}$ This version of the argument, differently from Schaffer's, does not need to assume Modal Exclusiveness or Necessity of Monism.

The argument from the possibility of emergence starts from the assumption that it is metaphysically possible that the cosmos has emergent properties. But a cosmos which has emergent properties is prior to its proper parts. Therefore, it is possible that the cosmos is fundamental. This conclusion, together with Modal Exclusiveness, entails that this cosmos is fundamental (Schaffer 2010a, pp. 55f.). Within the present framework, the main premise of the argument has to be reformulated as the

\footnotetext{
9 Schaffer claims that "Either it is metaphysically necessary for the cosmos to be a fundamental whole, or it is metaphysically necessary for the cosmos (if it has proper parts) to be derivative" (Schaffer 2010a, p. 56): the parenthetical condition is required to exclude cases like that of a cosmos composed of exactly one elementary particle.

${ }^{10}$ Of course, there is a sense in which even priority monism is threatened by the eventuality of "bad news" from physics: if those gunky hypotheses were conclusively refuted, even the argument from the epistemic possibility of gunk would be undermined. However, that would not show that priority monism is false, whereas the confirmation of those hypotheses would show that pluralism is false. The asymmetry is still in favour of priority monism over pluralism.
} 
claim that in some possible world there is a cosmos which has emergent properties, which seems a plausible assumption. But that is not sufficient to warrant that this cosmos could have emergent properties even if, as a matter of fact, has none, since it does not entail that there is a possible holistic cosmos which is identical to this cosmos. Actually, according to the view that I have outlined, a cosmos with no emergent property has such a character essentially. Therefore, there is no way to conclude that this cosmos is essentially fundamental from the mere possibility of cosmic emergence.

It seems that the argument from the possibility of emergence cannot be vindicated within the present framework. However, this argument plays a minor role in the overall strategy of Schaffer, who observes that such an argument “... lurks behind..." the argument from quantum mechanics (Schaffer 2010a, p. 55): once we know empirically that this cosmos, being an entangled system, has emergent properties, we do not need to invoke a priori the mere possibility of cosmic emergence anymore.

In conclusion, defenders of priority monism should be happy with giving up Necessity of Monism for Essentiality of Fundamentality, which offers significant gains at a small cost. The conjunction of priority monism and Essentiality of Fundamentality eludes Steinberg's objection and, once worked out by endorsing the Bigelow-Ellis-Lierse view of the cosmos as one of a kind, provides us with a theory which preserves the spirit of Necessity of Monism without imposing any arbitrary restriction on the space of metaphysical possibilities. On the other hand, most of Schaffer's arguments for priority monism, namely the argument from parsimony (Schaffer 2007), the argument from quantum mechanics (Schaffer 2010a), the argument from internal relatedness (Schaffer 2010b), and the argument from fundamental laws (Schaffer 2013), stand unaffected by this move, whereas the argument from the metaphysical possibility of gunk can be reshaped as an argument from the epistemic possibility of gunk. The only price to pay is to accept that the resulting version of priority monism is not supported by the argument from the possibility of emergence anymore, but we have seen that this is a negligible cost: all things considered, defenders of priority monism should pay it to buy the advantages offered by the replacement of Necessity of Monism with Essentiality of Fundamentality.

Acknowledgments I thank an anonymous referee for some helpful suggestions.

Open Access This article is distributed under the terms of the Creative Commons Attribution 4.0 International License (http://creativecommons.org/licenses/by/4.0/), which permits unrestricted use, distribution, and reproduction in any medium, provided you give appropriate credit to the original author(s) and the source, provide a link to the Creative Commons license, and indicate if changes were made.

\section{References}

Bigelow, J., Ellis, B., \& Lierse, C. (1992). The world as one of a kind: Natural necessity and laws of nature. The British Journal for the Philosophy of Science, 43, 371-388.

Dehmelt, H. (1989). Triton,... electron,... cosmon,...: An infinite regression? Proceedings of the National Academy of Sciences, 86, 8618-8619. 
Georgi, H. (1989). Effective quantum field theories. In P. Davies (Ed.), The new physics (pp. 446-457). Cambridge: Cambridge University Press.

Kripke, S. A. (1980). Naming and necessity. Cambridge, MA: Harvard University Press.

Plantinga, A. (1974). The nature of necessity. Oxford: Clarendon Press.

Schaffer, J. (2007). From nihilism to monism. Australasian Journal of Philosophy, 85, 175-191.

Schaffer, J. (2010a). Monism: The priority of the whole. Philosophical Review, 119, 31-76.

Schaffer, J. (2010b). The internal relatedness of all things. Mind, 119, 341-376.

Schaffer, J. (2013). The action of the whole. Proceedings of the Aristotelian Society, 87, 67-87.

Steinberg, A. (2015). Priority monism and part/whole dependence. Philosophical Studies, 172, 2025-2031. 